\title{
A Continuous Fiber Distribution Material Model for Human Cervical Tissue
}

\author{
Kristin M. Myers ${ }^{\mathrm{a}, 1}$, Christine P. Hendon ${ }^{\mathrm{b}}$, Yu Gan ${ }^{\mathrm{b}}$, Wang Yao ${ }^{\mathrm{a}}$, Kyoko \\ Yoshida $^{\mathrm{a}}$, Michael Fernandez ${ }^{\mathrm{a}}$, Joy Vink ${ }^{\mathrm{c}}$, Ronald J. Wapner ${ }^{\mathrm{c}}$ \\ ${ }^{a}$ Department of Mechanical Engineering, Columbia University School of Engineering and \\ Applied Science, New York, NY, U.S.A. \\ ${ }^{b}$ Department of Electrical Engineering, Columbia University School of Engineering and \\ Applied Science, New York, NY, U.S.A. \\ ${ }^{c}$ Department of Obstetrics and Gynecology, Columbia University Medical Center, New \\ York, NY, U.S.A.
}

\begin{abstract}
The uterine cervix during pregnancy is the vital mechanical barrier which resists compressive and tensile loads generated from a growing fetus. Premature cervical remodeling and softening is hypothesized to result in the shortening of the cervix, which is known to increase a womans risk of preterm birth. To understand the role of cervical material properties in preventing preterm birth, we derive a cervical material model based on previous mechanical, biochemical and histological experiments conducted on nonpregnant and pregnant human hysterectomy cervical tissue samples. In this study we present a three-dimensional fiber composite model that captures the equilibrium material behavior of the tissue in tension and compression. Cervical tissue is modeled as a fibrous composite material, where a single family of preferentially aligned and continuously distributed collagen fibers are embedded in a compressible neo-Hookean ground substance. The total stress in the collagen solid network is calculated by integrating the fiber stresses, and the shape of the fiber distribution is described by an ellipsoid where semi-principal axis lengths are fit to optical coherence tomography measurements. The composite material model is fit to averaged mechanical testing data from uni-axial compression and tension experiments,
\end{abstract}

\footnotetext{
${ }^{1}$ Corresponding Author: 500 W. 120th Street, Mudd 220, New York, NY 10027, email: kmm2233@columbia.edu
}

Preprint submitted to Journal of Biomechanics special issue on Reproductive BiomechanicsFebruary 20, 2015 
and averaged material parameters are reported for nonpregnant and term pregnant human cervical tissue. The model is then evaluated by investigating the stress and strain state of a uniform thick-walled cylinder under a compressive stress with collagen fibers preferentially aligned in the circumferential direction. This material modeling framework for the equilibrium behavior of human cervical tissue serves as a basis to determine the role of preferentially-aligned cervical collagen fibers in preventing cervical deformation during pregnancy.

Keywords: constitutive modeling, cervix, pregnancy, collagen fibers

\section{Introduction}

During pregnancy, a closed firm cervix is required to maintain the developing fetus in utero. Towards the end of gestation, a normal cervix significantly deforms, dilates and shortens in preparation for delivery. An early onset of cervical shortening, hypothesized to be caused by premature cervical remodeling, is known to put pregnant patients at risk for preterm birth (PTB) (Iams et al. 2011). The true causes of preterm birth remain to be determined, but the mechanical failure of the cervix is a final common pathway for multiple etiologies (Gravett et al., 2010; Solomon and Iams, 2014). The overall research goal of our work is to quantify the mechanical environment of pregnancy and to determine how the cervix functions as a resistive barrier to prevent PTB. The objectives of this study are to present an equilibrium constitutive material model that captures the tension/compression nonlinearity of previously published uniaxial data of human cervical tissue samples (Myers et al., 2008, 2010) and to describe how to incorporate directionality and dispersion parameters measured by optical coherence tomography (OCT) (Gan et al. 2014) into this material model. The purpose of this paper is to report fiber composite material properties for human cervical tissue and initiate a starting point for a 3-dimensional material model that can capture the salient nonlinear features of the cervical material behavior while accounting for its collagen fiber directionality.

The in vivo three-dimensional (3D) deformation and stress state of the cervix 
during pregnancy is complex due to patient-specific pelvic geometry, pelvic tissue material properties, passive and active external loading, and the contact and boundary conditions between organs (Fernandez et al., 2015; House et al. 2009, 2012, 2013, Mahmoud et al. 2013). The stress state of the cervix has been studied using CAD-based (House et al., 2012, Mahmoud et al., 2013) and patient-specific MRI-based (Fernandez et al., 2015) finite element models of the pregnant pelvis. Simulation results reveal that under a constant intrauterine pressure the stress state of the cervix is a combination of tension and compression (Fig. 1), where the magnitude and directionality of the principal stress and strain highly depend on the placement of the cervix within the pelvis, material characteristics of the cervix, and the interactions between the amniotic sac, the lower uterine segment, and the internal os (e.g. the opening of the cervix into the uterus) (Fernandez et al., 2015, House et al., 2012). Generally, there is a principal compressive stress in the longitudinal axis of the cervix, parallel to the inner canal, caused by the amniotic sac pushing on the internal os. This compressive stress translates into prominent tensile principal strains in the circumferential and radial directions. Taken together these forces cause the cervix to expand radially and shorten, similar to a thick-walled cylinder resisting a compressive axial stress.

The collagen ultrastructure of the cervix plays a crucial role to withstand the circumferential, radial, and longitudinal stresses throughout the tissue. A traditional view of the preferred directionality of the collagen was elucidated by x-ray diffraction techniques by Aspden from an average of eight nonpregnant human cervices (Aspden, 1988). This view details three seamless zones of aligned collagen with an inner and outer zone of longitudinal fibers and a middle zone of circumferential fibers. Additionally, this study reports a level of dispersion around the main fiber direction. More recently, second harmonic generation (SHG) (Feltovich et al., 2012) and OCT data (Gan et al. 2014) of human cervical tissue specimens reveal a large band of circumferential fibers that extend to the outer radial edges of the cervix (Fig.2A). From these ultrastructure studies, it is clear that the dense core of the cervix contains bands of collagen fibers 
circling the inner canal preventing circumferential dilation of the cervix. The width of this band of circumferential collagen and also the amount of dispersion for both nonpregnant and pregnant tissue are still under investigation. As we continue to elucidate these possible regions of preferentially-aligned collagen fibers, we develop here a material model that directly incorporates collagen fiber directionality and dispersion from OCT data (Gan et al., 2014).

A critical element to evaluating the mechanical loading pattern on the cervix during pregnancy is a micro-structurally inspired constitutive material model for cervical tissue that captures its material response to physiologically-relevant loading patterns. Previous mechanical testing data has found that the material response of human cervical tissue to loading is nonlinear, anisotropic, and timedependent, where the overall material response for pregnant tissue is orders of magnitude less than nonpregnant tissue (Badir et al., 2013; Fernandez et al. 2013: Hee et al., 2014; Liao et al., 2014, Myers et al., 2008, 2010, Parra-Saavedra et al., 2011; Yao et al., 2014). In both the instantaneous and equilibrium response, one of the most important features of cervical material behavior is the non-linearity between the tension and compression stress response. Cervical tissue is much softer in compression than tension. This asymmetry is due in part to the collagen fiber network, where collagen fibers support loads when they are engaged in a tensile strain.

The goal of this modeling study is to determine the simplest form of a material constitutive equation that describes the salient tension/compression feature of the cervical tissue's equilibrium stress response, after the transient visco- and poro-elastic effects have subsided. Additionally, the derivation considers the dominant features of the cervical extracellular matrix that are hypothesized to remodel and cause cervical softening during pregnancy, mainly the collagen fiber stiffness, directionality, and dispersion (Akins et al., 2010, Danforth, 1983, 1947 Feltovich et al. 2010, Gan et al., 2014, Myers and Ateshian, 2014, Myers et al. 2009 Read et al. 2007; Yoshida et al., 2014). We model cervical tissue as a composite material where the stress in the tissue is balanced by a continuouslydistributed collagen fiber network embedded in an isotropic compressible neo- 
Hookean ground substance (Lanir, 1983). The model contains a single family of preferentially oriented fibers, where the fibers are dispersed about the main direction described by an ellipsoidal orientation density function (Ateshian et al. 2009 Myers and Ateshian, 2014). Both directionality and dispersion are informed by OCT collagen fiber measurements of an axial slice of a nonpregnant and pregnant human cervical sample (Gan et al., 2014). We present the tension and compression uni-axial model fits to previously published data (Myers et al. 2008,2010 ). We illustrate how the cervical collagen ultrastructure plays a role in preventing cervical deformation by investigating the compression of a thickwalled cylinder with circumferentially aligned fibers, which serves as a simplistic toy model for the stress state of the cervix experienced in vivo.

\section{Methods}

\subsection{Equilibrium Material Model for Human Cervical Tissue}

A tissue material element of the cervix is modeled as a composite of a preferentially-aligned collagen network embedded within an isotropic, compressible neo-Hookean ground substance. Here we assume isothermal and equilibrium conditions, where the transient effects of the interstitial fluid pressurization and the intrinsic viscoelasticity of the collagen network have subsided. Additionally, in the initial form of this model we assume the tissue does not actively contract and that the fixed charge density of the entangled glycosaminoglycans is sufficiently low that it does not contribute to the compressive material response of the tissue. The collagen fiber network has a single family of aligned fibers, where the directionality and dispersion are informed by a measure of the ultrastructure of collagen fibers (Gan et al., 2014). The collagen fibers are extensible, deform affinely with the ground substance, and in the current form of the model they do not interact with each other.

The total Cauchy stress $\sigma^{\mathrm{TOT}}$ for this equilibrium cervical material model is given by

$$
\sigma^{\mathrm{TOT}}=\frac{1}{\mathrm{~J}} \frac{\partial \Psi^{\mathrm{TOT}}\left(\mathbf{F}, \hat{\mathbf{a}}_{\mathbf{o}}, \frac{b}{a}\right)}{\partial \mathbf{F}}(\mathbf{F})^{\mathrm{T}}
$$


where $\Psi^{\mathrm{TOT}}$ is the total Helmholtz free energy density of the continuously distributed collagen fiber composite, defined per unit reference volume. $\mathbf{F}$ is the deformation gradient given by a standard definition derived from the motion of the material from the reference to the deformed configuration, $\mathrm{J}=\operatorname{det} \mathbf{F}$ is the Jacobian, and $\hat{\mathbf{a}}_{\mathbf{o}}$ and $\frac{b}{a}$ are the preferred direction of a single family of collagen fibers and a measure of the fiber dispersion (per Eq.6) in the reference configuration, respectively. This hyperelastic relation is derived from thermodynamic restrictions imposed by the axiom of entropy inequality (Holzapfel, 2000).

The total Helmholtz free energy density $\Psi^{\text {TOT }}$ is the additive contribution from the fibrous collagen network $\Psi^{\mathrm{COL}}$ and the neo-Hookean ground substance $\Psi^{\mathrm{GS}}$ of non-collagenous ECM components, such as elastin, glycosaminoglycans, and other matricellular proteins, given by

$$
\Psi^{\mathrm{TOT}}\left(\mathbf{F}, \hat{\mathbf{a}}_{\mathbf{o}}, \frac{b}{a}\right)=\Psi^{\mathrm{GS}}(\mathbf{F})+\Psi^{\mathrm{COL}}\left(\mathbf{F}, \hat{\mathbf{a}}_{\mathbf{o}}, \frac{b}{a}\right) .
$$

The free energy density of the ground substance $\Psi^{\mathrm{GS}}$ is given by a standard isotropic, compressible neo-Hookean relation,

$$
\Psi^{\mathrm{GS}}=\frac{\mu}{2}\left(\mathrm{I}_{1}-3\right)-\mu \ln \mathrm{J}+\frac{\lambda}{2}(\ln J)^{2}
$$

where $\mathrm{I}_{1}=\operatorname{tr} \mathbf{C}$ is the first invariant of the right Cauchy-Green tensor $\mathbf{C}=$ $(\mathbf{F})^{\mathrm{T}} \mathbf{F}$ and $\mu$ and $\lambda$ are the standard lamé constants. These lamé constants combine to form the Young's modulus and Poisson's ratio of the ground substance $E^{\mathrm{GS}}=\frac{\mu\left(3+\frac{2 \mu}{\lambda}\right)}{1+\frac{\mu}{\lambda}}$ and $\nu^{\mathrm{GS}}=\frac{1}{2\left(1+\frac{\mu}{\lambda}\right)}$, respectively.

The free energy density of the solid collagen network $\Psi^{\mathrm{COL}}$ is an orientation average of the free energy density of a single collagen fiber $\Psi_{n}^{\mathrm{COL}}\left(\mathrm{I}_{\mathrm{n}}\right)$ (Eq. 5 . weighted by an ellipsoidal distribution $D\left(\mathbf{n}_{\mathbf{o}}, \frac{b}{a}\right)$ (Eq.6. and is given by

$$
\Psi^{\mathrm{COL}}\left(\mathbf{F}, \hat{\mathbf{a}}_{\mathbf{o}}, \frac{b}{a}\right)=\int_{0}^{2 \pi} \int_{0}^{\pi} H\left(I_{n}-1\right) \Psi_{n}^{\mathrm{COL}}\left(\mathrm{I}_{\mathrm{n}}\right) D\left(\mathbf{n}_{\mathbf{o}}, \frac{b}{a}\right) \sin \phi d \phi d \theta
$$

where $\mathbf{n}_{\mathbf{o}}=\cos \theta \sin \phi \mathbf{e}_{1}+\sin \theta \sin \phi \mathbf{e}_{2}+\cos \phi \mathbf{e}_{3}$ in a local Cartesian basis $\left\{\mathbf{e}_{1}, \mathbf{e}_{2}, \mathbf{e}_{3}\right\}$ with spherical coordinates $\theta$, and $\phi$ and $\hat{\mathbf{a}}_{\mathbf{o}}$ defines the preferred directionality. $I_{n}=\mathbf{n}_{\mathbf{o}} \cdot \mathbf{C} \cdot \mathbf{n}_{\mathbf{o}}$ is the square of the fiber stretch, and $H$ is the unit step function that enforces collagen fibers sustain only tension. The 
free energy density of a single fiber bundle $\Psi_{n}^{\mathrm{COL}}$ (i.e. collection of crosslinked collagen fibrils) is constitutively prescribed to be

$$
\Psi_{n}^{\mathrm{COL}}=\xi\left(\mathrm{I}_{\mathrm{n}}-1\right)^{\beta},
$$

where $\xi$ and $\beta$ represent material properties of the collagen fiber bundle. $\xi$ is the elastic modulus of the collagen fiber, with units of stress, and $\beta$ is a unitless stiffening parameter giving the shape of tensile nonlinearity of the fiber. (An odd value of $\beta$ is invalid if the unit step function $H$ is not employed in Equation (4).

The distribution of the single family of preferentially aligned collagen fibers is described by an ellipsoidal fiber distribution (Fig.2E), expressed in a local material element basis $\left\{\mathbf{e}_{i}\right\}$ using spherical coordinates:

$$
D\left(\mathbf{n}_{\mathbf{o}}, \frac{b}{a}\right)=\left(\frac{\cos ^{2} \theta \sin ^{2} \phi}{a^{2}}+\frac{\sin ^{2} \theta \sin ^{2} \phi}{b^{2}}+\frac{\cos ^{2} \phi}{b^{2}}\right)^{-\frac{1}{2}},
$$

where $[a, b]$ are the semi-axes of a planar ellipse fit (Fig.2D) to the collagen fiber orientation distribution (Fig.2C) normalized by,

$$
1=\int_{0}^{2 \pi} \int_{0}^{\pi} D\left(\mathbf{n}_{\mathbf{o}}, \frac{b}{a}\right) \sin \phi d \phi d \theta .
$$

For the case of the cervix as a thick-walled cylinder, and based on our initial OCT fiber-tracking maps (Fig.2A\&B), â aligns with the circumferential direction represented by the local coordinate $\mathbf{e}_{1}$ (Fig.2F). Considering, the OCT dispersion measurements are taken on the cervical plane perpendicular to the inner canal, we need to make an assumption regarding dispersion in the orthogonal plane. Therefore, we assume that the dispersion is equal in both orthogonal directions of the main fiber direction. In the case of the circumferential fibers the dispersion is in the longitudinal and radial directions, where the ratio $\frac{b}{a}$ gives the amount of dispersion. When $\frac{b}{a}$ is equal to 1 the fibers are evenly spread around a unit sphere, representing a case with no preferential direction in the reference configuration. When $\frac{b}{a}$ tends towards 0 , the fibers are highly aligned, representing a limiting case with no dispersion. 


\subsection{Collagen Fiber Ultrastructure Characterization}

We recently established a collagen fiber tracking protocol for human cervical tissue using optical coherence tomography (OCT) (Gan et al., 2014). In that work, we describe a methodology to numerically assemble multiple volume sets of OCT data to produce collagen fiber maps of axial cervical slices, and we demonstrate the methodology on specimens from one nonpregnant (age 40) and one pregnant (age 29, 36 weeks of gestation) patient who underwent a hysterectomy. Both samples were taken within $5 \mathrm{~mm}$ of the internal os. The OCT signal was conditioned based on a previously published algorithm for heart tissue (Fleming et al. 2008, Gan and Fleming, 2013) and optimized for cervical collagen (Gan et al. 2014).

In this paper, fiber orientation distributions (Fig. $2 \mathrm{C}$ ) were extracted from the OCT data within 12 different $1 \mathrm{~mm}$ x $1 \mathrm{~mm}$ regions: 3 different radial zones in the anterior, posterior, left, and right of the axial slice (measurement locations indicated in Fig.2 A \& B). Within each measurement location the prominent direction $\hat{\mathbf{a}}_{\mathbf{o}}$ was identified and the planar fiber dispersion was described by an ellipse (Eq.6), with $\phi=\frac{\pi}{2}$ and $\theta$ representing the angle from the preferred direction. The ellipse parameters $[a, b]$ were optimized using a nonlinear least squares optimization routine in Matlab (v 2013b) such that the equation described OCT dispersion data (Fig.2D). To extrapolate to a 3D ellipsoid, both the minor axes of the ellipsoid were set equal to $[b]$. Lastly, the major $[a]$ and minor $[b]$ semi-principal axes were normalized under the condition in Eq.7 (Fig. 2E).

\subsection{Model Fits to Uni-axial Mechanical Testing Data}

To obtain averaged material parameters $\left[E_{\mathrm{GS}}, \nu_{\mathrm{GS}}, \xi, \beta\right]$ the proposed material model was fit to equilibrium compression and tension data of nonpregnant and pregnant human cervical tissue reported in two previously published studies (Myers et al., 2008, 2010). The detailed mechanical testing protocols were published in those reports. Briefly, tissue samples were acquired from nonpregnant and term pregnant hysterectomy patients of various obstetric backgrounds and 
ages. Uni-axial tension and compression samples were taken from axial slices that were cut perpendicular to the inner canal at various locations throughout the cervix. Tensile strips ( $\sim 10 \mathrm{~mm}$ by $5 \mathrm{~mm})$ (Myers et al. 2010$)$ and compression cylindrical biopsies ( $\sim \varnothing 8 \mathrm{~mm}$ by $5 \mathrm{~mm})$ (Myers et al. 2008$)$ were cut exclusively from the dense mid-stromal region. All samples were tested in a hydrated environment in a series of ramp-hold tests. Equilibrium data were averaged here for all nonpregnant and pregnant samples after the transient material response has dissipated. The averaged data along with the range in the stress response are reported in Figure 4. Because it was challenging to obtain whole cervices during pregnancy, there was only a single set of pregnant tensile data reported, and therefore a range of tensile behavior is not reported.

Best-fit material parameters for these data were found by informing the material model with the collagen directionality $\left[\hat{\mathbf{a}}_{\mathbf{o}}\right]$ and averaged dispersion $\left[\frac{b}{a}\right]$ measured from our initial OCT results (Figure2 \& Table 1). We assumed that the preliminary OCT data was representative of typical nonpregnant and pregnant collagen fiber orientation and dispersion (This assumption will be tested as we collect more OCT data, and the limitations of this assumption are discussed below). Therefore, since tension samples were mechanically tested with the loading axis aligned with the circumferential direction and compression samples were tested with the loading axis aligned with the longitudinal axis (i.e. the inner canal axis), models fits were done such that $\hat{\mathbf{a}}_{\mathbf{o}}$ aligned accordingly. To get a first approximation of the material properties for the ground substance $\left[E_{\mathrm{GS}}, \nu_{\mathrm{GS}}\right]$, an analytical form of the material model with no fiber dispersion was fit to the tension and compression stress response data using a nonlinear least squares optimization routine in Matlab (v 2013b). Once these parameters were obtained from the simplified model, the full form of the model with the collagen dispersion was fit to the data using FEBio (v2.0, http://febio.org/), where the ellipsoidal fiber distribution model is numerically implemented and documented (Ateshian et al. 2009). In this last step of the fitting, the collagen fiber parameters $[\xi, \beta]$ were optimized by inspection such that the model calculations matched the experimental data. 


\subsection{Thick-Walled Cylinder Investigation}

The deformation patterns of a thick-walled cylinder under compressive loading were investigated to understand how changes in the material parameters, particularly the fiber ultrastructure, affect the ability of the structure to carry a load. This simplistic boundary value problem attempts to explore the contribution of material characteristics as opposed to the complex geometric factors present during pregnancy. The in vivo loading conditions of the cervix depend on many interacting geometric and material factors, include anatomical shape, tissue material properties, and contact between the organs. A review of these variables and the current state of pregnancy biomechanics is presented in a companion paper in this special issue (Myers et al., 2015).

A thick-walled cylinder was constructed within FEBio using dimensions measured from MRI data of a pregnant patient at 22 weeks of gestation as reported in (Fernandez et al., 2015). The inner canal radius was $8 \mathrm{~mm}$, the outer radius of the cervix was $28 \mathrm{~mm}$, and the length was $38 \mathrm{~mm}$. The model was meshed using 3510 8-node hexahedral elements, where each element was assigned the proposed ellipsoidal dispersion material model. The preferential direction of the collagen fibers $\left[\hat{\mathbf{a}}_{\mathbf{o}}\right]$ was aligned with the circumferential direction of the cylinder, with equal amounts of dispersion in the radial and longitudinal directions (Fig. $2 \mathrm{~F}$ ). A longitudinal $1 \mathrm{kPa}$ compressive stress was applied to the top of the cylinder, with sliding boundary conditions on the bottom and stress-free conditions on the outer walls and inner canal (Fig. $2 \mathrm{~F}$ ). These boundary conditions attempt to mimic the stress pattern seen in the posterior part of the cervix in mid-pregnancy (Fig.11). The longitudinal, radial, and circumferential strains within the cylinder were investigated as a function of cylinder fiber modulus $\xi$ and fiber dispersion $\frac{b}{a}$, where the baseline material parameters for comparison were set equal to the averaged nonpregnant values reported in Table 1 . 


\section{Results}

\subsection{Cervical Collagen Ultrastructure by OCT: Directionality \& Dispersion}

The detailed OCT methodology and analysis of cervical collagen directionality and dispersion are reported in (Gan et al. 2014), and the preliminary results are used here to demonstrate how to incorporate the OCT data into the continuous fiber distribution material model. OCT fiber maps of axial slices of human cervix indicate a large band of circumferential collagens in nonpregnant (Fig.2A) and pregnant (Fig.2B) cervix, where the circumferential fibers exist as far as the outer radial edges of the cervix. Similar to the x-ray data, the collagen fibers are dispersed about the main fiber direction in both the nonpregnant and pregnant tissue (Fig. 3). Averaging the dispersion $\left(\frac{b}{a}\right)$ data for 12 OCT measurement locations for each of the cervical slices from the internal os, the collagen fiber dispersion is higher in the pregnant tissue slice when compared to the nonpregnant tissue slice (Fig. 3 \& Table 1 . This result was not statistically significant, and there was also no statistically significant difference between collagen dispersion measured in the different radial zones (Student's t-test, $\mathrm{p}>0.05$; two-tailed power $=0.025$ with $\mathrm{p}=0.05$; Fig. 3). An increase in sample size is needed to conclude if collagen fiber dispersion changes throughout pregnancy and if there are distinct zones of fiber families in the human cervix.

\subsection{Fiber Composite Model Fit to Uni-axial Data}

The proposed fiber composite material model, informed by the averaged OCT directionality and dispersion (Fig. 3), captured the uni-axial tension and compression equilibrium stress behavior of the cervical tissue (Fig. 4). The bestfit material properties for the averaged set of nonpregnant and pregnant data are in Table 1. With a single set of material parameters, one each for the nonpregnant and the pregnant case, the material model describes the averaged tension and compression uni-axial stress response (Fig.4). The total sums of the squared differences between model calculations and data (calculated in $\mathrm{kPa}$ ) are 212 and 0.120 for the nonpregnant and pregnant fit, respectively. 
The range in the collagen fiber modulus $\xi$ reported in Table 1 for the nonpregnant case captures the highest and lowest tensile stress response reported (Fig.4A), while all other material parameters are kept to their averaged value. Changing $\xi$ alone, however, does not sufficiently capture the highest compressive stress response for the nonpregnant tissue (Fig. $4 \mathrm{~B}$ ). The range in the material parameter $E_{G S}$ reported in Table1 for the pregnant case captures the highest and lowest compressive stress response (Fig.4D), while all other material parameters are kept to their averaged value.

\subsection{Compression of a Thick-walled Cylinder with Circumferential Fibers}

For a thick-walled cylinder under a $1 \mathrm{kPa}$ compressive longitudinal load, where $\hat{\mathbf{a}}_{\mathbf{o}}$ is aligned circumferentially, and with material properties as listed for nonpregnant tissue in Table1, the magnitude of radial strain (Fig.5A) is larger than the circumferential strain (Fig.5B). The collagen fiber modulus $\xi$ influences the magnitude of the radial and circumferential strain experienced within the cylinder, but it does not influence the pattern of strain. The radial strain is mostly uniform throughout the thickness ( $<3 \%$ change throughout thickness), whereas the circumferential strain is nonuniform. The radial strain ranges from 0.027 to 0.067 , for the highest and lowest measured values of the collagen fiber modulus $\xi$, respectively. The corresponding circumferential strain is the smallest at the inner canal $(0.022-0.053)$ and is the largest at the outer edge $(0.025$ 0.060). As the fiber modulus $\xi$ increases, and the overall bulk behavior of the tissue gets stiffer, there is a protective effect of the collagen fibers to maintain the radius and thickness of the thick-walled structure under a constant load. Incremental improvements to this protective effect decreases as $\xi$ continues to increase because of the nonlinear stiffening of the material behavior.

Preferentially-aligned fibers in the circumferential direction help maintain the radius of a thick-walled cylinder under a constant compressive load (Fig.6). Decreasing the dispersion such that the collagen fibers become more circumferentially aligned causes a reduction to the circumferential strain and an increase in the radial strain. In other words, the pattern of circumferential strain evolves 
with decreasing fiber dispersion, where an increase in anisotropy creates regions of reduced strain closer to the inner canal. There is an approximately $30 \%$ reduction in circumferential strain at the inner canal if all of the collagen fibers within the cylinder have the lowest measured dispersion in the nonpregnant tissue $(\mathrm{b} / \mathrm{a}=0.58)$ as compared to a cylinder with the randomly distributed fibers $(\mathrm{b} / \mathrm{a}=1)$. The level of radial strain, which tends to change the cylinder thickness, only increases by $3 \%$ for this level of alignment $(b / a=0.58)$.

\section{Discussion}

Collagen fiber ultrastructure is a key component to the mechanical function of the human cervix during pregnancy. As such, we present a 3D constitutive material model for human cervical tissue that captures its material response to physiologically-relevant loading patterns and is equipped with ultrastructural parameters that describe cervical softening during pregnancy. Experimental uni-axial compression and tension tests reveal that the stress response of the tissue in tension is much higher than in compression and that pregnant tissue is considerably softer than nonpregnant tissue (Myers et al., 2008, 2010), and imaging data of the cervical stroma reveal zones of preferentially-aligned collagen fibers with a level of dispersion (Aspden, 1988, Gan et al., 2014). To describe these known material characteristics of the cervical tissue, we develop here a fiber composite material model with a continuously distributed fiber network, where changes to the collagen fiber stiffness capture the drastic softening that occurs during pregnancy (Fig.4).

During pregnancy there is a drastic softening that must occur within the cervical tissue to accommodate the delivery of the baby (see cervical mechanics review in this special issue (Myers et al., 2015)). This softening is accomplished through a complex biochemical cascade of events that remodel the ECM components of the tissue (Holt et al., 2011, Read et al., 2007, Word et al., 2007). Our initial evidence from collagen ultrastructure imaging suggests that the overall preferential direction of the collagen fibers remains in the circumferential di- 
rection, but the level of dispersion increases (Gan et al., 2014). This increase in dispersion alone cannot capture the substantial softening that occurs during pregnancy. To capture the difference in the nonpregnant and pregnant material stress response the collagen fiber $\xi$ modulus must be reduced by approximately two orders of magnitude (from an average of 190 to $0.7 \mathrm{kPa}$ ). Therefore, we hypothesize this reduction for the individual fiber component indicates that along with changes to the collagen ultrastructure, the collagen must intrinsically remodel to accommodate such a level of cervical softening. Evidence of this collagen remodeling has been found in human and mouse tissue. Previous studies by our group have shown that the amount of collagen content does not significantly change in pregnancy, in both human (Myers et al. 2009) and mouse tissue (Yoshida et al., 2014). Instead tissue solubility increases in human pregnant tissue, indicating a reduction in collagen crosslinking (Myers et al. 2009). In mouse tissue we found evidence of a drastic decline in the total mature crosslink density (moles of pyridinoline and deoxypyridinoline normalized by moles of collagen) in day 15 pregnant tissue compared to day 6 pregnant tissue, of a 19 day mouse gestational period (Yoshida et al. 2014). To prove our hypothesis, we are currently measuring the collagen crosslinking density in nonpregnant (Zork et al. 2014) and pregnant human cervical tissue.

The nonlinearity in the tension/compression stress response is typical for soft hydrated tissues with a collagen fiber network, where collagen fibers provide resistance to tension and any preferential alignment will give added deformation resistance. In the case of the cervix, the collagen fibers have a preferred circumferential orientation with a level of dispersion, as viewed by x-ray diffraction (Aspden, 1988) and OCT (Gan et al., 2014) investigations. This collagen ultrastructure plays a key role in maintaining the mechanical function of the cervix during pregnancy. In the first form of the model, we ignore the transient material response of the tissue to investigate the contribution of fiber stiffness, fiber directionality, and dispersion. We demonstrate this mechanical function here using a thick-walled cylinder under a compressive load and in patientspecific finite element models presented in (Fernandez et al., 2015). 
For the case of the thick-walled cylinder, the circumferential collagen fibers reduce the amount of circumferential strain, where decreasing levels of dispersion increase this resistance to changes in the radius. We hypothesize that this added resistance to the circumferential strain is a protective feature of the cervix preventing dilation and over-stretching of interspersed cells that may control cervical remodeling. Additionally, the level of fiber distribution and dispersion aids in deformation resistance in directions that are not aligned with the main fiber family. This dispersion plays an important role in resisting deformation for the non-uniform and asymmetric loading found in the physiologic-loading scenario, where the geometry of the anatomy and the placement of the cervix within the pelvis drives patient-specific patterns of cervical stress and strain (Fernandez et al. 2015). A previous study, by Liao et al., details a three-fiber family model (with no dispersion) to describe the cervical collagen ultrastructure (Liao et al. 2014), using a modeling method common in arterial wall mechanics (Gasser et al., 2006). This material model does a good job of capturing the mechanical response of the cervical tissue to inflation of the inner canal (Liao et al., 2014). However, it is unknown if this type of three-fiber family model can capture the tension/compression behavior seen in the in-vivo loading scenario. A full comparison of all cervical material models are given in a cervical mechanics review presented this special issue (Myers et al. 2015).

Obtaining and testing human cervical tissue is a challenge, especially for pregnant tissue specimens. In this modeling study we made simplifying assumptions based on the available data sets for human cervical tissue to establish a framework to study the cervical collagen ultrastructure. These assumptions create limitations, where components of our model need to be validated and extended. First, the OCT, tensile, and compression data were not conducted on the same cervical tissue. Here, we use initial imaging data results coupled with a database of averaged stress responses from previous studies. Second, previous mechanical tests reveal that the material response of human cervical tissue is time-dependent, with stress-relaxation and hysteresis measured during ramp-hold and load-unload tests (Myers et al., 2008, 2010, Yao et al., 2014). 
The next iteration of our model will account for this viscoelastic, and possible poroelastic behavior (Fernandez et al., 2013), by extending a previously explored one-dimensional nonlinear viscoelastic model (Myers et al., 2010) to 3D. Third, we have not mechanically tested human cervical tissue in tension in multiple anatomical directions and we have not taken compression tests to high levels of strain in which fiber directionality influences the results. We are currently conducting additional mechanical tests to validate if this fiber dispersion model can predict tissue behavior in multiple anatomical directions. Lastly, the thickwalled cylinder is modeled as being homogeneous with even levels of dispersion and directionality throughout the radius. Here we do not account for the possibility of additional families of fibers directed longitudinally. The heterogeneity of tissue characteristics and its effect on how the cervix maintain a mechanical load will be a topic of another study.

\section{Conclusion}

Here we present a necessary framework for a 3D equilibrium material model that accounts for the collagen fiber ultrastructure of the tissue. Equipping the material model with features of the collagen network allows us to explore the influence of individual collagen fiber properties and network organization to the overall mechanical response of the tissue. As we continue to collect and mechanically test cervical tissue specimens, we will add deformation mechanisms to the constitutive model as needed to describe the deformation as a result of physiologically-relevant loading scenarios and to describe the remodeling process of the cervix in pregnancy. In this study, we show that a continuouslydistributed fiber network embedded in a compressible ground substance can represent the stress response of the tissue to uni-axial loading, and we demonstrate that the collagen directionality and dispersion play a role in resisting physiological relevant deformation during pregnancy. 
426 Acknowledgements

${ }_{427}$ Research reported in this publication was supported in part by the the Na${ }_{428}$ tional Science Foundation (BRIGE1125670 \& EEC-1342273). The content is 429 solely the responsibility of the authors and does not necessarily represent the 430 official views of the National Science Foundation. 


\section{References}

Akins, M., Luby-Phelps, K., Mahendroo, M., 2010. Second harmonic generation imaging as a potential tool for staging pregnancy and predicting preterm birth. J Biomed Opt 15, 026020.

Aspden, R., 1988. Collagen organization in the cervix and its relation to mechanical function. Coll Relat Res 8, 103-112.

Ateshian, G., Rajan, V., Chahine, N., Canal, C., Hung, C., 2009. Modeling the Matrix of Articular Cartilage Using a Continuous Fiber Angular Distribution Predicts Many Observed Phenomena. J Biomech Eng 131, 061003.

Badir, S., Bajka, M., Mazza, E., 2013. A novel procedure for the mechanical characterization of the uterine cervix during pregnancy. J Mech Behav Biomed Mater 27, 143-153.

Danforth, D., 1983. The morphology of the human cervix. Clin Obstet Gynecol 26, 7-13.

Danforth, D.N., 1947. The fibrous nature of the human cervix, and its relation to the isthmic segment in gravid and nongravid uteri. Am J Obstet Gynecol $53,541-560$.

Feltovich, H., Hall, T., Berghella, V., 2012. Beyond cervical length: emerging technologies for assessing the pregnant cervix. Am J Obstet Gynecol , 1-43.

Feltovich, H., Nam, K., Hall, T.J., 2010. Quantitative ultrasound assessment of cervical microstructure. Ultrason Imaging 32, 131-142.

Fernandez, M., House, M., Jambawalikar, S., Vink, J., Wapner, R., Zweben, N., Myers, K., 2015. Investigating the Mechanical Function of the Cervix during Pregnancy using Finite Element Models derived from High Resolution 3D MRI. in-review Comput Methods Biomech Biomed Engin . 
Fernandez, M., Vink, J., Yoshida, K., Wapner, R., Myers, K., 2013. Direct Measurement of the Permeability of Human Cervical Tissue. J Biomech Eng 135, 021024.

Fleming, C.P., Ripplinger, C.M., Webb, B., Efimov, I.R., Rollins, A.M., 2008. Quantification of cardiac fiber orientation using optical coherence tomography. J Biomed Opt 13, 030505.

Gan, Y., Fleming, C.P., 2013. Extracting three-dimensional orientation and tractography of myofibers using optical coherence tomography. Biomed Opt Express 4, 2150.

Gan, Y., Yao, W., Myers, K.M., Hendon, C.P., 2014. An automated 3d registration method for optical coherence tomography volumes, in: Engineering in Medicine and Biology Society (EMBC), 2014 36th Annual International Conference of the IEEE, IEEE. pp. 3873-3876.

Gasser, T., Ogden, R., Holzapfel, G., 2006. Hyperelastic modelling of arterial layers with distributed collagen fibre orientations. J R Soc Interface 3, 15-35.

Gravett, M.G., Rubens, C.E., Nunes, T.M., GAPPS Review Group, 2010. Global report on preterm birth and stillbirth (2 of 7$)$ : discovery science. BMC Pregnancy Childbirth 10 Suppl 1, S2.

Hee, L., Liao, D., Sandager, P., Gregersen, H., Uldbjerg, N., 2014. Cervical Stiffness Evaluated In Vivo by Endoflip in Pregnant Women. PLoS ONE 9, e91121.

Holt, R., Timmons, B., Akgul, Y., Akins, M., Mahendroo, M., 2011. The molecular mechanisms of cervical ripening differ between term and preterm birth. Endocrinology 152, 1036-1046.

Holzapfel, G.A., 2000. Nonlinear Solid Mechanics: A Continuum Approach for Engineering. John Wiley \& Sons. 
House, M., Bhadelia, R., Myers, K., Socrate, S., 2009. Magnetic resonance imaging of three-dimensional cervical anatomy in the second and third trimester. Eur J Obstet Gynecol Reprod Biol 144 Suppl 1, S65-9.

House, M., Feltovich, H., Hall, T., Stack, T., Patels, A., Socrate, S., 2012. Three-dimensional, extended field-of-view ultrasound method for estimating large strain mechanical properties of the cervix during pregnancy. Ultrason Imaging 34, 1-14.

House, M., McCabe, R., Socrate, S., 2013. Using imaging-based, threedimensional models of the cervix and uterus for studies of cervical changes during pregnancy. Clinical anatomy (New York, N.Y.) 26, 97-104.

Iams, J.D., Cebrik, D., Lynch, C., Behrendt, N., Das, A., 2011. The rate of cervical change and the phenotype of spontaneous preterm birth. Am J Obstet Gynecol 205, 130.e1-130.e6.

Lanir, Y., 1983. Constitutive equations for fibrous connective tissues. J Biomech $16,1-12$.

Liao, D., Hee, L., Sandager, P., Uldbjerg, N., Gregersen, H., 2014. Identification of biomechanical properties in vivo in human uterine cervix. J Mech Behav Biomed Mater 39, 27-37.

Mahmoud, H., Wagoner Johnson, A., Chien, E.K., Poellmann, M.J., McFarlin, B., 2013. System-Level Biomechanical Approach for the Evaluation of Term and Preterm Pregnancy Maintenance. J Biomech Eng 135, 021009.

Myers, K., Ateshian, G., 2014. Interstitial growth and remodeling of biological tissues: Tissue composition as state variables. J Mech Behav Biomed Mater $29,544-556$.

Myers, K., Feltovich, H., Mazza, E., Vink, J., Bajka, M., Wapner, R., Hall, T., House, M., 2015. The Mechanical Role of the Cervix In Pregnancy. accepted to J of Biomech Special Edition of Reproductive Biomechanics . 
Myers, K., Paskaleva, A., House, M., Socrate, S., 2008. Mechanical and biochemical properties of human cervical tissue. Acta Biomater 4, 104-116.

Myers, K., Socrate, S., Paskaleva, A., House, M., 2010. A study of the anisotropy and tension/compression behavior of human cervical tissue. J Biomech Eng $132,021003$.

Myers, K., Socrate, S., Tzeranis, D., House, M., 2009. Changes in the biochemical constituents and morphologic appearance of the human cervical stroma during pregnancy. Eur J Obstet Gynecol Reprod Biol 144 Suppl 1, S82-9.

Parra-Saavedra, M., Gómez, L., Barrero, A., Parra, G., Vergara, F., Navarro, E., 2011. Prediction of preterm birth using the cervical consistency index. Ultrasound Obstet Gynecol 38, 44-51.

Read, C.P., Word, R.A., Ruscheinsky, M.A., Timmons, B.C., Mahendroo, M.S., 2007. Cervical remodeling during pregnancy and parturition: molecular characterization of the softening phase in mice. Reprod 134, 327-340.

Solomon, C., Iams, J., 2014. Prevention of Preterm Parturition. N Engl J Med. $370,254-261$.

Word, R.A., Li, X., Hnat, M., Carrick, K., 2007. Dynamics of cervical remodeling during pregnancy and parturition: mechanisms and current concepts. Semin Reprod Med 25, 69-79.

Yao, W., Yoshida, K., Fernandez, M., Vink, J., Wapner, R., Ananth, C., Oyen, M., Myers, K., 2014. Measuring the compressive viscoelastic mechanical properties of human cervical tissue using indentation. J Mech Behav Biomed Mater $34,18-26$.

Yoshida, K., Jiang, H., Kim, M., Vink, J., Cremers, S., Paik, D., Wapner, R., Mahendroo, M., Myers, K., 2014. Quantitative Evaluation of Collagen Crosslinks and Corresponding Tensile Mechanical Properties in Mouse Cervical Tissue during Normal Pregnancy. PLoS ONE 9, e112391. 
Zork, N.M., Myers, K.M., Yoshida, K., Cremers, S., Jiang, H., Ananth, C.V., Wapner, R.J., Kitajewski, J., Vink, J., 2014. A systematic evaluation of collagen cross-links in the human cervix. Am J Obstet Gynecol , 1-8.

\section{List of Tables}

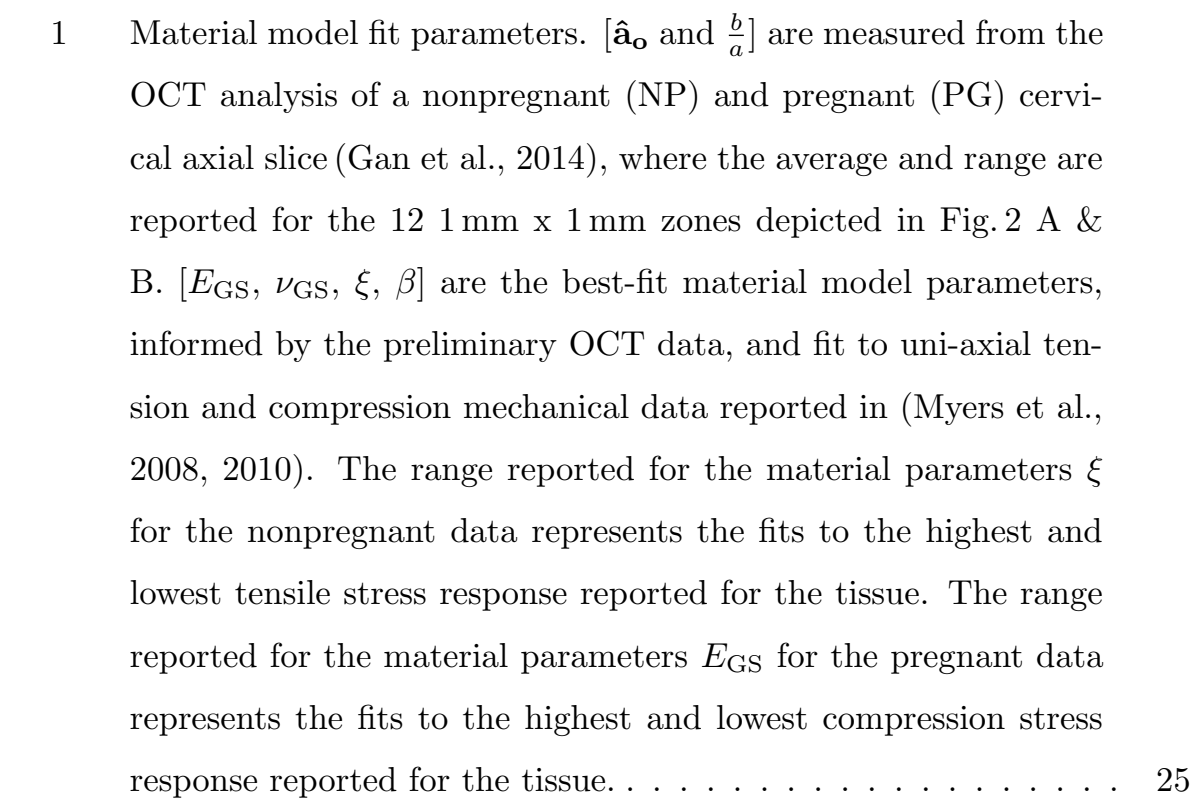

\section{List of Figures}

\begin{tabular}{l}
$11 \quad$ Representative principal strain and stress directions depicted for \\
a pregnant patient at 20 weeks of gestation. The intrauter- \\
ine pressure of $8.6 \mathrm{kPa}$ was applied to the inner surface of the \\
fetal membranes, which are adhered to the lower uterine seg- \\
ment (Fernandez et al. $[2015)$. The mechanical deformation state \\
of the human cervix is a mix of tension and compression. De- \\
pending on the placement of the cervix within the pelvis, regions \\
of the cervix experience a longitudinal compressive stress. . . . . 26 \\
\hline
\end{tabular}




\begin{tabular}{|c|c|c|c|}
\hline 4 & 2 & Optical coherence tomography (OCT) fiber orientation maps and & \\
\hline 65 & h & measurement locations for an axial slice of A) a nonpregnant and & \\
\hline & & B) a pregnant human cervix. C) A typical shape of the fiber & \\
\hline 67 & & distribution for a $1 \mathrm{~mm} \times 1 \mathrm{~mm}$ zone. D) A planar ellipse fit to & \\
\hline 88 & & the fiber distribution shown in C. E) An extrapolation and nor- & \\
\hline 69 & & malization (Eq. 7) of the planar ellipse into a 3D ellipsoid where & \\
\hline 70 & & it is assumed the minor radii are equal. F) Thick-walled cylin- & \\
\hline 571 & & der loading condition, with sliding boundary conditions on the & \\
\hline 72 & Б & bottom and stress-free conditions on the outer walls and inner & \\
\hline 573 & & canal. & 27 \\
\hline 574 & 口 & Fiber dispersion $\frac{b}{a}$ in the outer, middle, and inner zones for the & \\
\hline 575 & 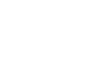 & nonpregnant and pregnant tissue axial slice. There is in an in- & \\
\hline 576 & 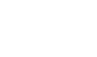 & crease in dispersion for the pregnant tissue slice compared to the & \\
\hline 577 & 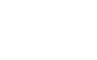 & nonpregnant slice. There was no statistically significant differ- & \\
\hline 578 & & ence in fiber dispersion between the zones for each slice. . & \\
\hline 579 & 口 & A) Averaged tension and B) compression equilibrium stress in & \\
\hline 580 & 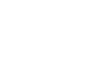 & response to uni-axial loading for nonpregnant tissue. C) Aver- & \\
\hline 581 & 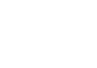 & aged tension and D) compression equilibrium stress in response & \\
\hline 582 & L & to uni-axial loading for nonpregnant tissue. Material model fits & \\
\hline 583 & 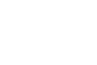 & are presented for the averaged data and the range when possible, & \\
\hline 584 & 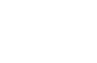 & $\begin{array}{l}\text { and material parameters are reported in Table } \\
\end{array}$ & \\
\hline 585 & 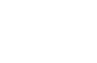 & tension and compression mechanical data are from (Myers et al., & \\
\hline 586 & & {$[2008[2010] \ldots \ldots \ldots$} & 29 \\
\hline 587 & 5 & Effect of collagen fiber modulus $\xi$. An increase in $\xi$ decreases & \\
\hline 588 & b & the amount of radial and circumferential strain in a thick-walled & \\
\hline 589 & 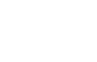 & cylinder that maintains a compressive stress of $1 \mathrm{kPa}$. The mate- & \\
\hline 590 & 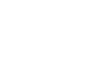 & rial properties used for this simulation are listed for the nonpreg- & \\
\hline 591 & 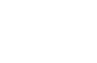 & nant tissue in Table 1 , where the range represents the material & \\
\hline 592 & h & model fit to the highest and lowest tensile stress response mea- & \\
\hline 593 & & $\begin{array}{l}\text { sured for nonpregnant human cervical tissue (Myers et al., 2010). } \\
\end{array}$ & \\
\hline
\end{tabular}


$694 \quad$ Effect of collagen fiber dispersion $\frac{b}{a}$. A decrease in collagen fiber

595 dispersion, corresponding to an increase in circumferential fiber

596 alignment, increases the amount of radial strain and decreases the

597 amount of circumferential strain in a thick-walled cylinder that

$598 \quad$ maintains a compressive stress of $1 \mathrm{kPa}$. The material properties

$599 \quad$ used for this simulation are listed for the nonpregnant tissue in

600 Table|1| where the range in dispersion represents the range in

601 dispersion measured for a nonpregnant sample (Gan et al., 2014).

602 The results indicated in the box represent the average material

603 characteristics for nonpregnant tissue. . . . . . . . . . . . . . . . 31 


\begin{tabular}{|c||c|c|c|c|c|c|}
\hline $\mathbf{N P} / \mathbf{P G}$ & $E_{\mathrm{GS}}[\mathrm{kPa}]$ & $\nu_{\mathrm{GS}}$ & $\xi[\mathrm{kPa}]$ & $\beta$ & $\hat{\mathbf{a}}_{\mathbf{o}}$ & $\frac{b}{a}$ \\
\hline \hline $\mathbf{N P}$ & 2 & 0.3 & $190(40-460)$ & 3 & circum & $0.77(0.58-0.90)$ \\
\hline $\mathbf{P G}$ & $0.65(0.50-0.80)$ & 0.3 & 0.7 & 2 & circum & $0.84(0.64-0.98)$ \\
\hline
\end{tabular}

Table 1: Material model fit parameters. [ $\hat{\mathbf{a}}_{\mathbf{o}}$ and $\left.\frac{b}{a}\right]$ are measured from the OCT analysis of a nonpregnant (NP) and pregnant (PG) cervical axial slice (Gan et al. 2014, where the average and range are reported for the $121 \mathrm{~mm} \times 1 \mathrm{~mm}$ zones depicted in Fig.2 A \& B. [ $E_{\mathrm{GS}}, \nu_{\mathrm{GS}}, \xi$, $\beta]$ are the best-fit material model parameters, informed by the preliminary OCT data, and fit to uni-axial tension and compression mechanical data reported in (Myers et al., 2008, 2010). The range reported for the material parameters $\xi$ for the nonpregnant data represents the fits to the highest and lowest tensile stress response reported for the tissue. The range reported for the material parameters $E_{\mathrm{GS}}$ for the pregnant data represents the fits to the highest and lowest compression stress response reported for the tissue. 


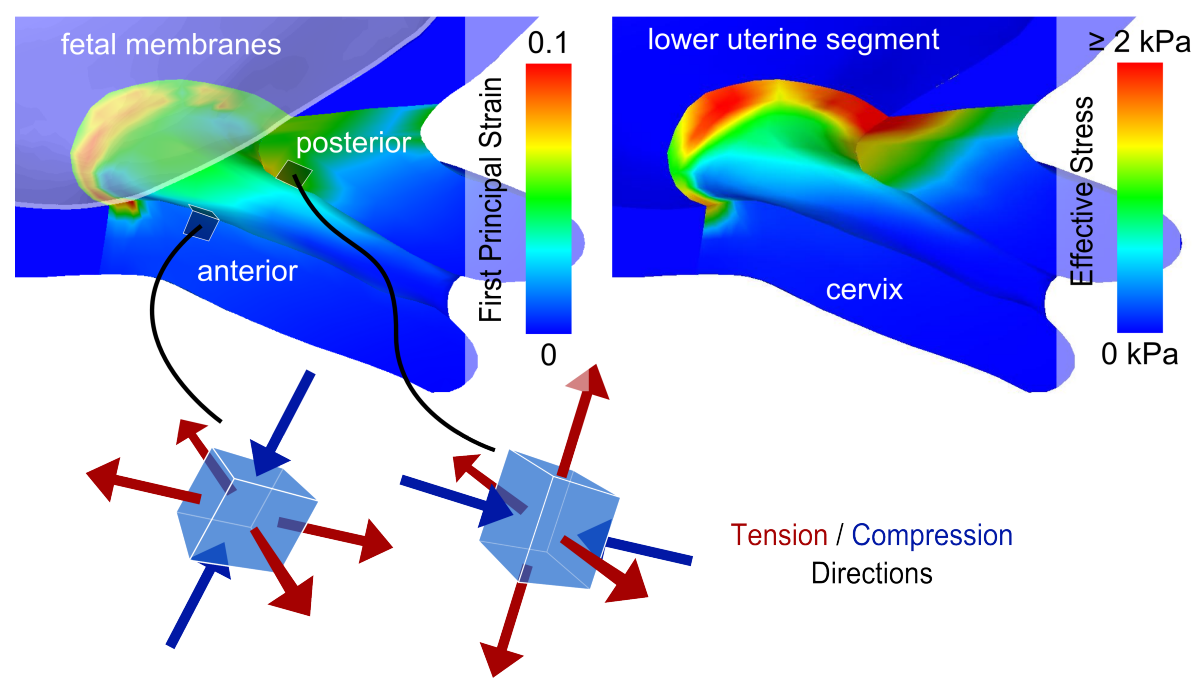

Figure 1: Representative principal strain and stress directions depicted for a pregnant patient at 20 weeks of gestation. The intrauterine pressure of $8.6 \mathrm{kPa}$ was applied to the inner surface of the fetal membranes, which are adhered to the lower uterine segment Fernandez et al. 2015). The mechanical deformation state of the human cervix is a mix of tension and compression. Depending on the placement of the cervix within the pelvis, regions of the cervix experience a longitudinal compressive stress. 

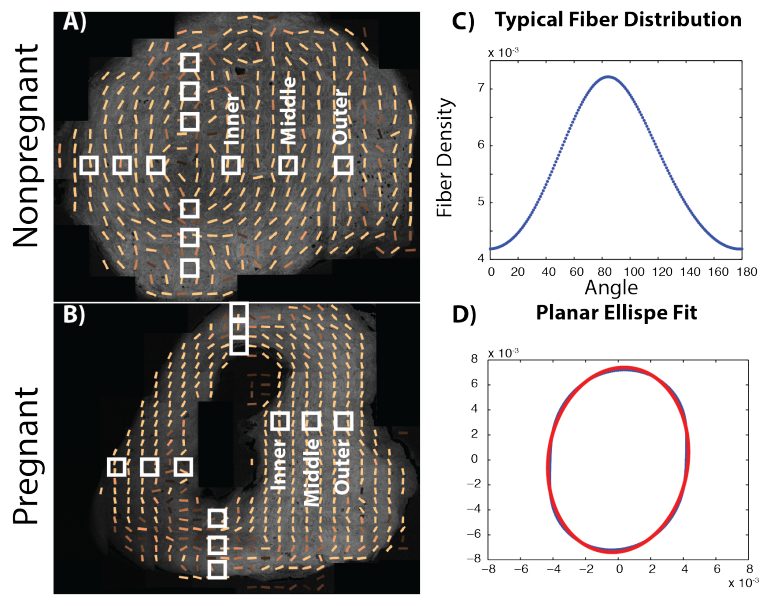

\section{E) 3D Ellipsoidal Extrapolation \\ \& Normalization}

D)
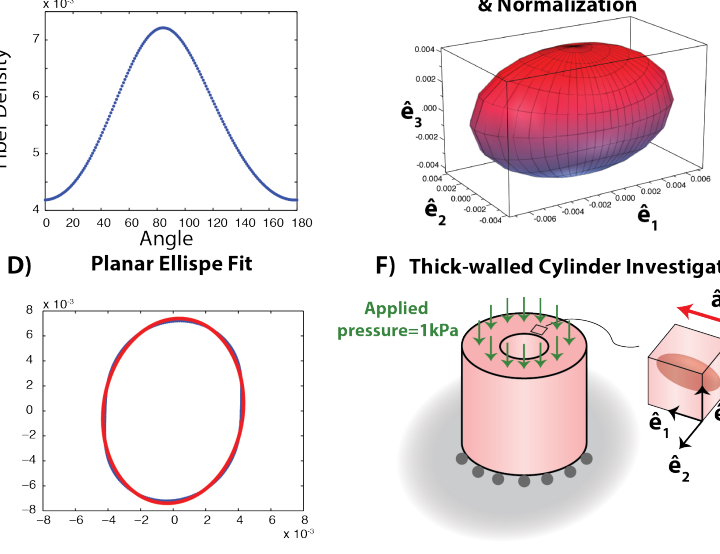

F) Thick-walled Cylinder Investigation

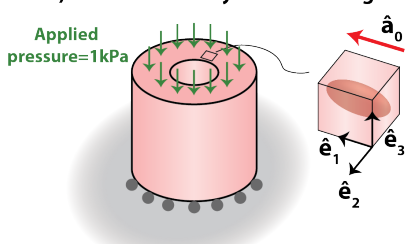

Figure 2: Optical coherence tomography (OCT) fiber orientation maps and measurement locations for an axial slice of A) a nonpregnant and B) a pregnant human cervix. C) A typical shape of the fiber distribution for a $1 \mathrm{~mm} \times 1 \mathrm{~mm}$ zone. D) A planar ellipse fit to the fiber distribution shown in C. E) An extrapolation and normalization (Eq.7) of the planar ellipse into a $3 \mathrm{D}$ ellipsoid where it is assumed the minor radii are equal. F) Thick-walled cylinder loading condition, with sliding boundary conditions on the bottom and stress-free conditions on the outer walls and inner canal. 

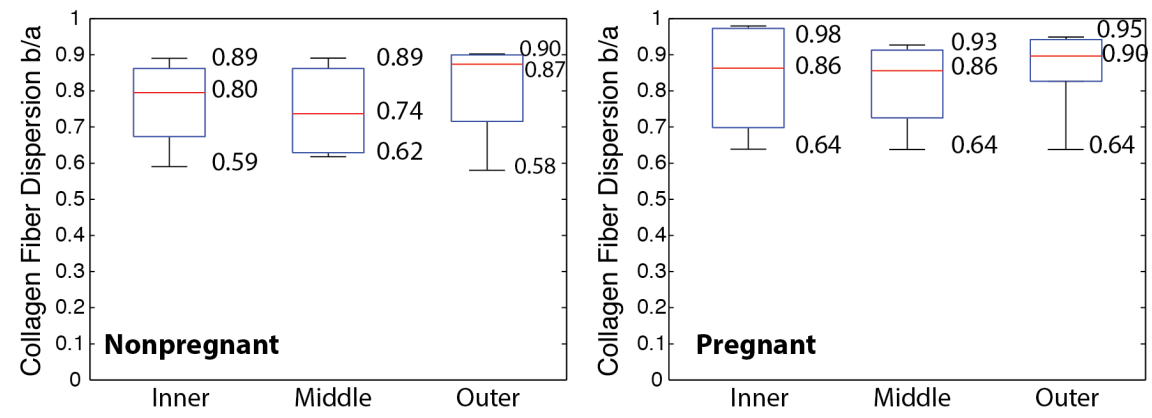

Figure 3: Fiber dispersion $\frac{b}{a}$ in the outer, middle, and inner zones for the nonpregnant and pregnant tissue axial slice. There is in an increase in dispersion for the pregnant tissue slice compared to the nonpregnant slice. There was no statistically significant difference in fiber dispersion between the zones for each slice. 

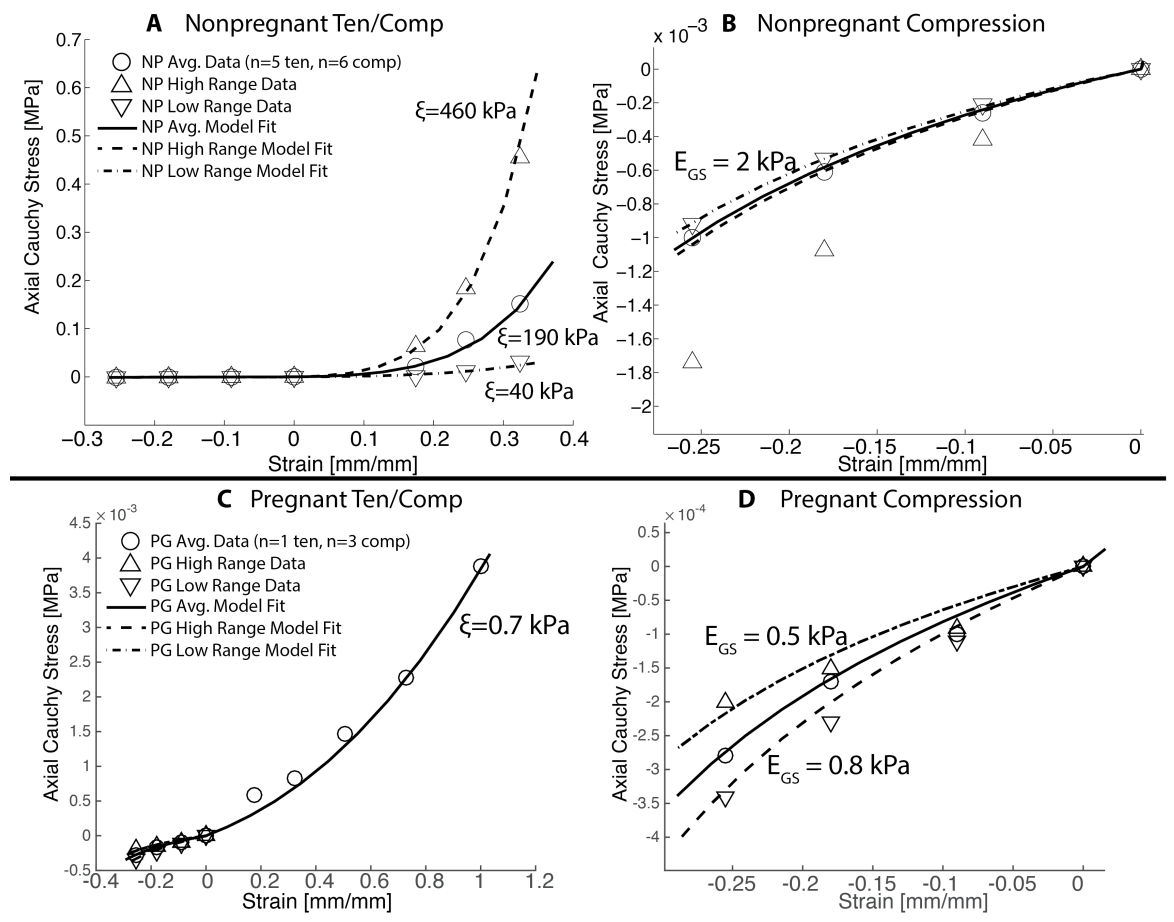

Figure 4: A) Averaged tension and B) compression equilibrium stress in response to uni-axial loading for nonpregnant tissue. C) Averaged tension and D) compression equilibrium stress in response to uni-axial loading for nonpregnant tissue. Material model fits are presented for the averaged data and the range when possible, and material parameters are reported in Table 1 The uni-axial tension and compression mechanical data are from (Myers et al. 2008, 2010) 


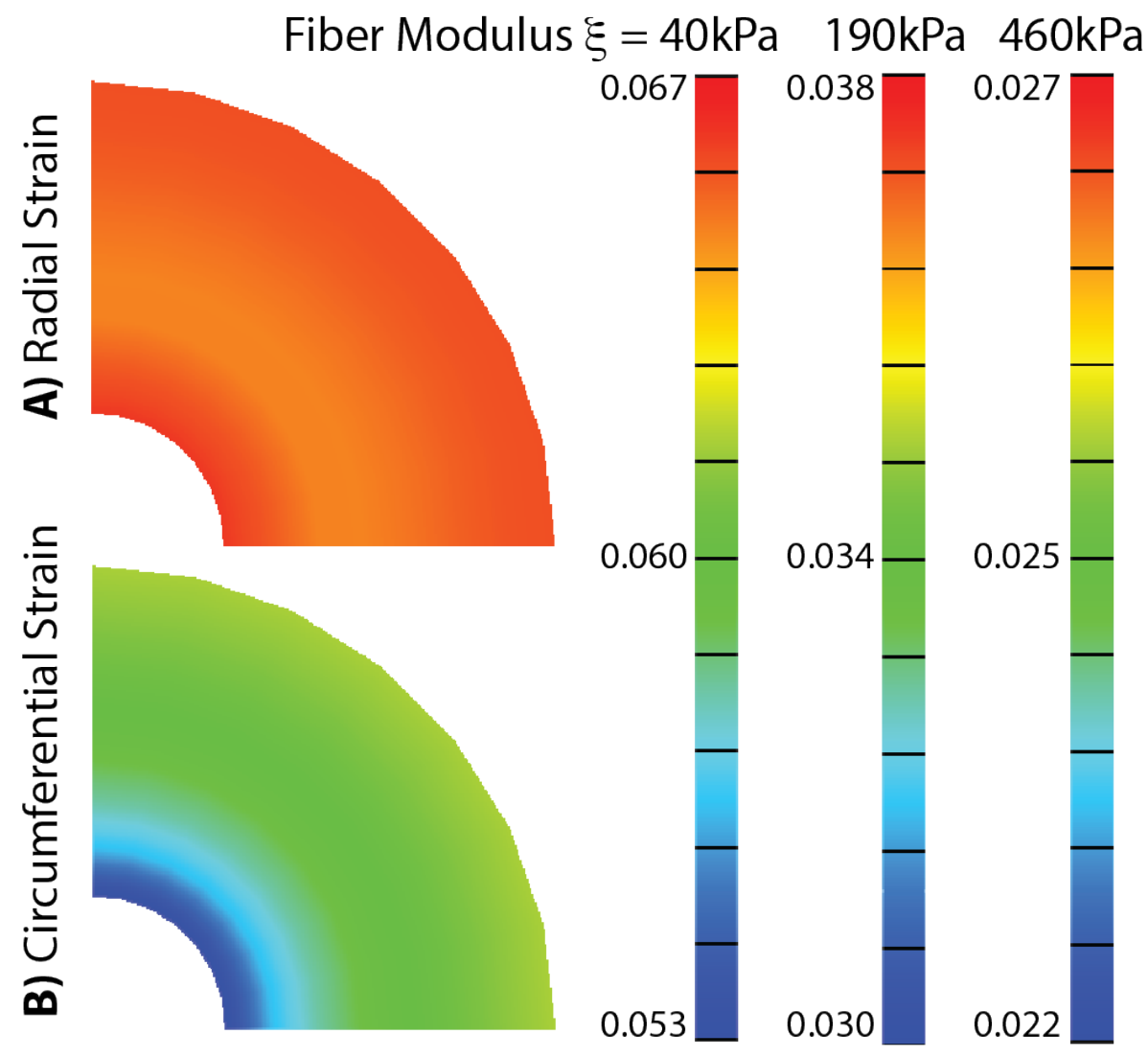

Figure 5: Effect of collagen fiber modulus $\xi$. An increase in $\xi$ decreases the amount of radial and circumferential strain in a thick-walled cylinder that maintains a compressive stress of $1 \mathrm{kPa}$. The material properties used for this simulation are listed for the nonpregnant tissue in Table 1 where the range represents the material model fit to the highest and lowest tensile stress response measured for nonpregnant human cervical tissue (Myers et al. 2010). 


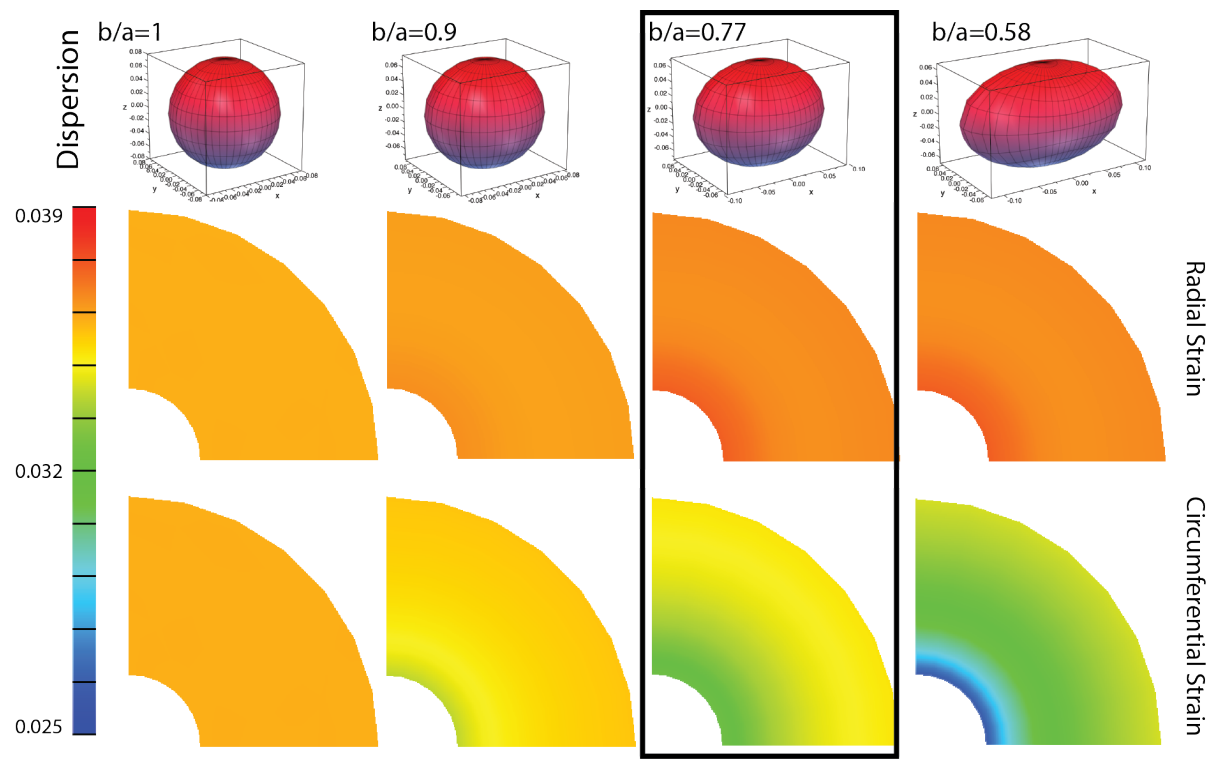

Figure 6: Effect of collagen fiber dispersion $\frac{b}{a}$. A decrease in collagen fiber dispersion, corresponding to an increase in circumferential fiber alignment, increases the amount of radial strain and decreases the amount of circumferential strain in a thick-walled cylinder that maintains a compressive stress of $1 \mathrm{kPa}$. The material properties used for this simulation are listed for the nonpregnant tissue in Table 1 where the range in dispersion represents the range in dispersion measured for a nonpregnant sample (Gan et al. 2014. The results indicated in the box represent the average material characteristics for nonpregnant tissue. 